\title{
The Effects of Air Vitiation on the Supersonic Turbulent Channel flow using Direct Numerical Simulation
}

\author{
Xiaoping Chen ${ }^{1}$ \\ Zhejiang Sci-Tech University, Hangzhou, Zhejiang, 310018, P. R. China \\ Xiaopeng $\mathrm{Li}^{2}$ \\ Institute of Mechanics, Chinese Academy of Sciences, Beijing, 100190, P. R. China \\ and \\ Hua-Shu DOU ${ }^{3}$ \\ Zhejiang Sci-Tech University, Hangzhou, Zhejiang, 310018, P. R. China
}

\begin{abstract}
Temporally evolving supersonic turbulent channel flows are simulated using direct numerical simulation (DNS) approach at Mach number 2.56, Reynolds number 7000 with water vapor $\left(\mathrm{H}_{2} \mathrm{O}\right)$ mass fraction from 0.00 to 0.161 to study the air vitiation effects. Then, the turbulent statistical characteristics and velocity-temperature correlations have been studied based on the DNS database. It is found that in fully developed turbulent channel flow, many of turbulent statistical characteristics used to express supersonic turbulent channel flow of pure air also hold for the $\mathrm{H}_{2} \mathrm{O}$ considered. The difference between fluctuating and turbulent Mach number points to a significant non zero velocitytemperature correlations. After a nondimensional static temperature parameters introduced, the mean velocity-temperature correlations collapses between current DNS results. The results of strong Reynolds analogy decrease with $\mathrm{H}_{2} \mathrm{O}$ mass fraction increasing, and modified strong Reynolds analogy show a better agreement than original strong Reynolds analogy. In addition, the correlation $\boldsymbol{R}_{u}$, $T$, isn't remained the same between the different $\mathrm{H} 2 \mathrm{O}$ mass fraction cases.
\end{abstract}

$\rho=$ density $\quad$ Nomenclature

\footnotetext{
${ }^{1}$ Doctor, Key Laboratory of Fluid Transmission Technology of Zhejiang Province, chenxp075@163.com.

${ }^{2}$ Doctor, State Key Laboratory of High Temperature Gas Dynamics, 1xpyfy@163.com.

${ }^{3}$ Professor, Key Laboratory of Fluid Transmission Technology of Zhejiang Province, huashudou@yahoo.com, AIAA fellow.
} 


$$
\begin{aligned}
& p \quad=\quad \text { pressure } \\
& T=\text { temperature } \\
& E \quad=\quad \text { total energy } \\
& h=\text { mixture enthalpy } \\
& c c \quad=\quad \text { sound speed } \\
& t=\text { time } \\
& x_{i}, x_{j}=\text { position vector }(i, j=1,3) \\
& \delta_{i, j}=\quad \text { Kronecker delta } \\
& u_{i}, u_{j}=\text { velocity vector } \\
& \sigma_{i j}=\text { shear stress tensor } \\
& S_{i j}, S_{k k}=\quad \text { strain rate tensor }(k=1,3) \\
& \mu=\text { mixture viscosity } \\
& \operatorname{Pr}=\text { Prandtl number } \\
& \text { Re }=\text { Reynolds number } \\
& \mathrm{Ma}=\text { Mach number } \\
& R=\text { universal gas constant } \\
& M_{s}=\text { component molecular weight }(s=1,3) \\
& Y_{s}=\text { component mass fraction } \\
& w \quad=\quad \text { wall conditions } \\
& \text { c }=\text { channel centerline } \\
& \text { ref }=\text { reference conditions } \\
& \text { * } \quad=\text { dimensional flow variable } \\
& +\quad=\quad \text { scaling with inner or wall values. } \\
& s \quad=\quad \text { specie variable } \\
& \{\cdot\}=\text { Favre average } \\
& =\text { turbulent fluctuations }
\end{aligned}
$$




\section{Introduction}

ROUND test experiment is crucial approach for supersonic flow study, which is the core topic for scramjet development. The combustion heating facility is widely used in scramjet ground test experiment to provide enough high enthalpy. However, a significant problem is that the high enthalpy air are seriously vitiated by several species, for example, $\mathrm{H}_{2} \mathrm{O}, \mathrm{CO}_{2}, \mathrm{CO}, \mathrm{H}, \mathrm{OH}, \mathrm{O}$, and $\mathrm{NO}$, which are not of representative or very few in actual atmosphere, so-called air vitiation relative to pure air [1]. The flow field in such air vitiation can be influenced by chemical and physical effects due to the different species from actual atmosphere. Therefore, the effect of air vitiation is one important study to the development of scramjet technologies.

Edelman et al. (1969) presents an analytical study of the effects of vitiation heating including equilibrium, vibrational and chemical relaxation, finite rate condensation, combustion, mixing, and over all test engine performance. The study is considered to be the starting study of air vitiation effects. Over the years, several more experimental studies have been reported in the literature to help understand air vitiation effects [3-8]. Pellett et al. (2002) offers a detailed review and analysis on the physics and chemistry of scramjet ignition and flame holding combustion processes, and the known effects of air vitiation on these processes. With the development of computational fluids dynamics (CFD), numerical simulations are widely used in the community. Recently, Ingenito (2015) have performed a CFD simulation of the combustor configuration to provide useful information in terms of efficiency at various vitiation percentages. Theoretical laws and remedies have been proposed for the ground to flight data extrapolation. Although many air vitiation effects have been studied, most of the works focus on the performance comparative analysis, and few works to investigate their influence on the flow field. However, the performance is mainly dependent on the flow flied, so it's necessary to study the mean and instantaneous flow flied affected by the air vitiation effects.

Due to direct numerical simulation (DNS) solves the Navier-Stokes equations directly and do not contains any modeling errors, it is become a powerful tool to study the flow mechanism. DNS of supersonic turbulent channel flows have been performed at Mach 1.5 and 3 by Coleman et al. (1995) and Huang et al. (1995). In order to deeply understand the supersonic turbulence flow, the effect of wall temperature [13-16], Mach number [17], and high enthalpy [18-19] have been detailed discussed. The results indicate that those parameters not only influence the average flow and instantaneous flow, but also turbulent structures and Reynolds analogy. The strong Reynolds analogy (SRA) is one of important factors in the supersonic turbulent flow, which is called the relationships between 
velocity and temperature fluctuations and can be used to instead of energy (temperature) equation in engineering application. The SRA suggested by Morkovin [20] is based on the assumption that the fluctuation in total temperature is nearly zero. In further developments that are based on considering the influence of the heat flux on the wall or eliminating the influence of the wall temperature, Cebeci et al. [21] employed a similarity relation between velocity and temperature to derive an extended form of SRA (ESRA). The check validity of classic and modified SRA for supersonic turbulent flow has also been performed by several researchers [15-18], while the air vitiation effects on the supersonic turbulent channel flow have not been understood clearly.

Chen et al. [22] performed a DNS of temporally evolving supersonic turbulent channel flow with $\mathrm{H}_{2} \mathrm{O}$ at Mach number 2.56 and Reynolds number $2.8 \times 10^{5}$. Based on this, a series DNS of supersonic turbulent channel flows are carried out under various $\mathrm{H}_{2} \mathrm{O}$ mass fractions to investigate the air vitiation effects. Then, the characteristics of the time averaged and fluctuating flow flied and instantaneous flow flied are assessed. Finally, detailed velocitytemperature correlations under various $\mathrm{H}_{2} \mathrm{O}$ mass fractions are studied.

\section{Governing Equations and Numerical Approach}

The governing equations are the time dependent three dimensional Navier-Stokes equations for a compressible fluid in nondimensional form.

$$
\begin{gathered}
\frac{\partial \rho}{\partial t}+\frac{\partial}{\partial x_{j}}\left(\rho u_{j}\right)=0 \\
\frac{\partial\left(\rho u_{i}\right)}{\partial t}+\frac{\partial}{\partial x_{j}}\left(\rho u_{i} u_{j}+p \delta_{i j}-\frac{1}{\operatorname{Re}_{r e f}} \sigma_{i j}\right)=0 \\
\frac{\partial E}{\partial t}+\frac{\partial}{\partial x_{j}}\left((E+p) u_{j}-\frac{1}{\operatorname{Re}_{r e f}}\left(u_{i} \sigma_{i j}+\frac{\mu C p}{\operatorname{Pr}} \partial T / \partial x_{j}\right)\right)=0
\end{gathered}
$$

where the total energy and the shear stress tensor are given by:

$$
\begin{gathered}
E=\rho h-p+\frac{1}{2} \rho u_{i} u_{i} \\
\sigma_{i j}=2 \mu S_{i j}-\frac{2}{3} \mu \delta_{i j} S_{k k}, \quad S_{i j}=\frac{1}{2}\left(\frac{\partial u_{i}}{\partial x_{j}}+\frac{\partial u_{j}}{\partial x_{i}}\right)
\end{gathered}
$$

In this paper, semiempirical formulas or curve fits will be used to simply the solving method of thermodynamic and transport properties. The species thermodynamic properties, including specific heat and enthalpy, are computed 
by NASA Glenn [23], while the species transport properties (viscosity) is taken from the work of CFD-ACE [24], which can be described as follows:

$$
\begin{gathered}
C p_{\mathrm{s}}=\frac{T_{r e f}{ }^{*}}{U_{r e f}{ }^{* 2}} \frac{R^{*}}{M_{s}^{*}}\left(a_{1} T^{*-2}+a_{2} T^{*-1}+a_{3}+a_{4} T^{*}+a_{5} T^{* 2}+a_{6} T^{* 3}+a_{7} T^{* 4}\right) \\
h_{s}=\frac{1}{U_{r e f}{ }^{*}} \frac{R^{*} T^{*}}{M_{s}^{*}}\left(-a_{1} T^{*-2}+a_{2} \ln T^{*} / T^{*}+a_{3}+a_{4} T^{*} / 2+a_{5} T^{* 2} / 3+a_{6} T^{* 3} / 4+a_{7} T^{* 5} / 6+b_{1} / T^{*}\right) \\
\mu_{s}=\frac{1}{\mu_{r e f}{ }^{*}} \frac{A T^{*} / 2}{B+T^{*}}
\end{gathered}
$$

Table 1 Nondimensional parameters for the supersonic turbulent channel flow

\begin{tabular}{ccccc}
\hline \hline$\rho_{\text {ref }}{ }^{*}, \mathrm{kgm}^{-3}$ & $U_{\text {ref }}{ }^{*}, \mathrm{~ms}^{-1}$ & $T_{\text {ref }}{ }^{*}, \mathrm{~K}$ & $\mathrm{Ma}_{\text {ref }}$ & $\mathrm{Re}_{\text {ref }}$ \\
\hline 0.208 & 1551.0 & 887.0 & 2.56 & 7000.0 \\
\hline \hline
\end{tabular}

Then, a common mixture rule for specific heat and enthalpy is JANAF rule [25], and mixture viscosity is calculated using Wilke's rule [26]. Table 1 gives the nondimensional parameters for the various $\mathrm{H}_{2} \mathrm{O}$ mass fractions, which include bulk velocity, wall density and temperature. The table also gives the Mach number Ma $\mathrm{ref}_{\text {f }}$ (based on the bulk velocity and sound speed at the walls) and Reynolds number $\mathrm{Re}_{\mathrm{ref}}$ (defined in terms of bulk velocity and channel half width, density and viscosity at the walls). Initial conditions are given by superimposing random velocity fluctuations upon a laminar parabolic velocity profile $\left(\left\{u_{1}\right\}=1.5\left(1-y^{2}\right),\left\{u_{2}\right\}=\left\{u_{3}\right\}=0\right)$ and uniform density and temperature fields $(\{\rho\}=0.9567,\{\mathrm{~T}\}=1.0451)$. The convection terms in the compressible Navier-Stokes equations are discretized by the $7^{\text {th }}$ order WENO scheme [27]. The viscous terms are approximated with an $8^{\text {th }}$ order central difference. A $3^{\text {th }}$ order Runge Kutta method is used for the time integration.

Table 2 Mass fraction, grid resolution and domain size for the supersonic turbulent channel flow

\begin{tabular}{cccccc}
\hline \hline Cases & $\mathrm{Y}_{\mathrm{O} 2}: \mathrm{Y}_{\mathrm{N} 2}: \mathrm{Y}_{\mathrm{H} 2 \mathrm{O}}$ & $L_{1} \times L_{2} \times L_{3}$ & $n_{1} \times n_{2} \times n_{3}$ & $\Delta x_{1}{ }^{+} \times \Delta x_{2 w}{ }^{+} \times \Delta x_{3}{ }^{+}$ & $\mathrm{Re}_{\tau}$ \\
\hline Case 1 & $0.306 \times 0.694 \times 0.000$ & & & $17.23 \times 0.31089 \times 8.62$ & 537.85 \\
Case 2 & $0.298 \times 0.675 \times 0.027$ & & $17.24 \times 0.31107 \times 8.62$ & 538.16 \\
Case 3 & $0.290 \times 0.656 \times 0.054$ & & & $17.03 \times 0.30720 \times 8.51$ & 531.45 \\
Case 4 & $0.282 \times 0.637 \times 0.081$ & $2 \pi \times 2 \times \pi$ & $196 \times 512 \times 196$ & $17.08 \times 0.30818 \times 8.54$ & 533.15 \\
Case 5 & $0.274 \times 0.618 \times 0.108$ & & & $16.99 \times 0.30647 \times 8.49$ & 530.20 \\
Case 6 & $0.266 \times 0.599 \times 0.135$ & & & $17.55 \times 0.31664 \times 8.78$ & 547.79 \\
Case 7 & $0.257 \times 0.582 \times 0.161$ & & & $17.57 \times 0.31697 \times 8.79$ & 548.37 \\
\hline \hline
\end{tabular}


The mixture gas is composed by the oxygen $\left(\mathrm{O}_{2}\right)$, nitrogen $\left(\mathrm{N}_{2}\right)$ and water vapor $\left(\mathrm{H}_{2} \mathrm{O}\right)$. We used a series DNS of supersonic turbulent channel flows with $\mathrm{H}_{2} \mathrm{O}$ mass fractions $\left(\mathrm{Y}_{\mathrm{H} 2 \mathrm{O}}\right)$ ranging from 0.000 to 0.161 . To isolate the effect of varying $\mathrm{H}_{2} \mathrm{O}$ mass fraction, the ratio $\mathrm{O}_{2}$ mass fraction $\left(\mathrm{Y}_{\mathrm{O} 2}\right)$ to $\mathrm{N}_{2}$ mass fraction $\left(\mathrm{Y}_{\mathrm{N} 2}\right.$ ) is kept constant, which can be seen from table 2. The domain size $\left(L_{1} \times L_{2} \times L_{3}\right)$, the grid size $\left(\Delta x_{1} \times \Delta x_{2} \times \Delta x_{3}\right)$, the number of grid points $\left(n_{1} \times n_{2} \times n_{3}\right)$ and wall Reynolds number $\left(R e_{\tau}\right)$ are also given in Table 2, respectively. In order to increase computational efficiency, a well-designed block-structured grid containing about $19.7 \mathrm{M}$ computational cells is applied. Uniform grids are used in the streamwise and spanwise directions, while geometrically stretched grids are used in the wall normal direction. Periodic boundary conditions have been used in the streamwise and spanwise directions. On the wall boundaries, nonslip conditions are used for three velocity components, and wall temperature is kept isothermal.

\section{Velocity and Temperature Statistics}

In order to study $\mathrm{H}_{2} \mathrm{O}$ mass fraction effects, the mean flow quantities need to be analyzed. Figure 1 plots some of mean flow parameters in wall normal direction, including streamwise velocity, static temperature and specific heat, respectively. It is can be seen that the mean static temperature and specific heat increase and mean streamwise velocity decrease away from the wall. The reason may be that shock waves and large kinetic dissipation make the kinetic energy transform to internal energy. It is indicated that the mean streamwise velocity collapse in wall normal direction, while the mean static temperature and specific heat decrease with $\mathrm{H}_{2} \mathrm{O}$ mass fraction increasing. In addition, the mean specific heat few smaller than its initial value, which relative deviates are all about $2.3 \%$.
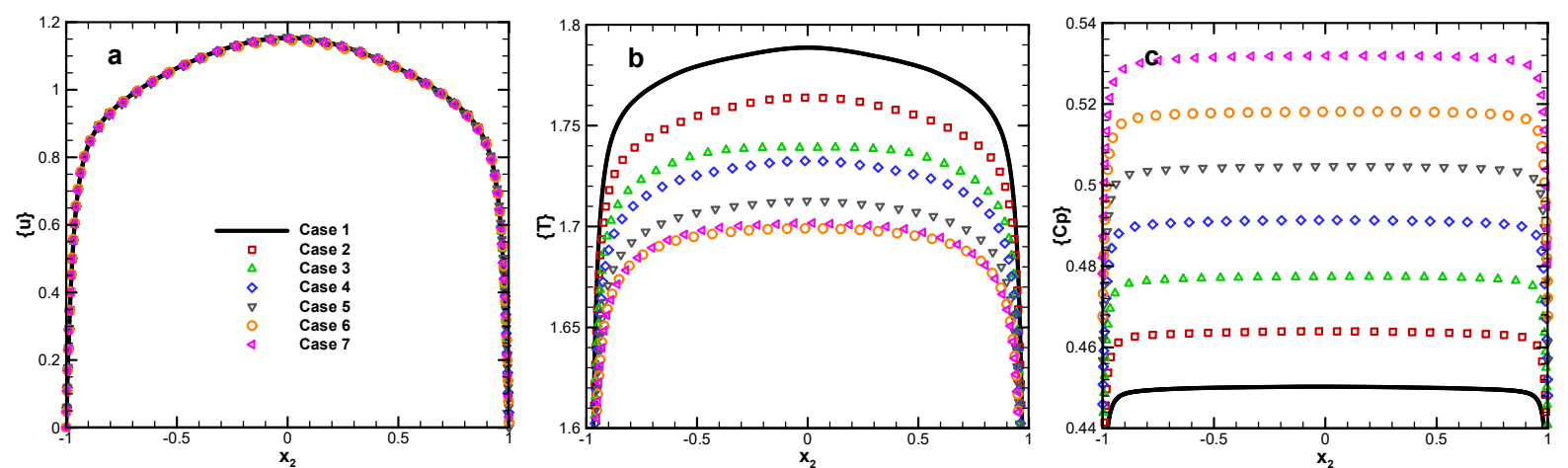

Fig.1 Distribution of the mean $a$ ) streamwise velocity, $b$ ) static temperature and $c$ ) specific heat.

The RMS (room mean square) fluctuation could response the importance of properly accounting for the turbulent intensity. RMS fluctuation of the streamwise velocity and static temperature are plotted in Fig.2. RMS fluctuation of 
streamwise velocity defined as $u_{\mathrm{rms}}^{\prime}=\sqrt{u^{\prime 2}}$ ( similar to $\mathrm{T}_{\mathrm{rms}}^{\prime}$ ). When normalized by conventional wall variables (defined in terms of the mean density, viscosity and shear stress at the wall), it is found that the differences are small. The relative large deviation occurs at the region of $0.96 \leq|y| \leq 0.70$, and the maximum deviation is less than $7.5 \%$. A small region in Fig.2 (a) is chosen and enlarged to display in Fig.2 (b), which show the deviations more clearly. Fairly large RMS fluctuation of static temperature is found, especially near the walls, where the maximum value is about $8 \%$. However, RMS fluctuation of static temperature is not collapsed the profiles for different $\mathrm{H}_{2} \mathrm{O}$ mass fraction cases.
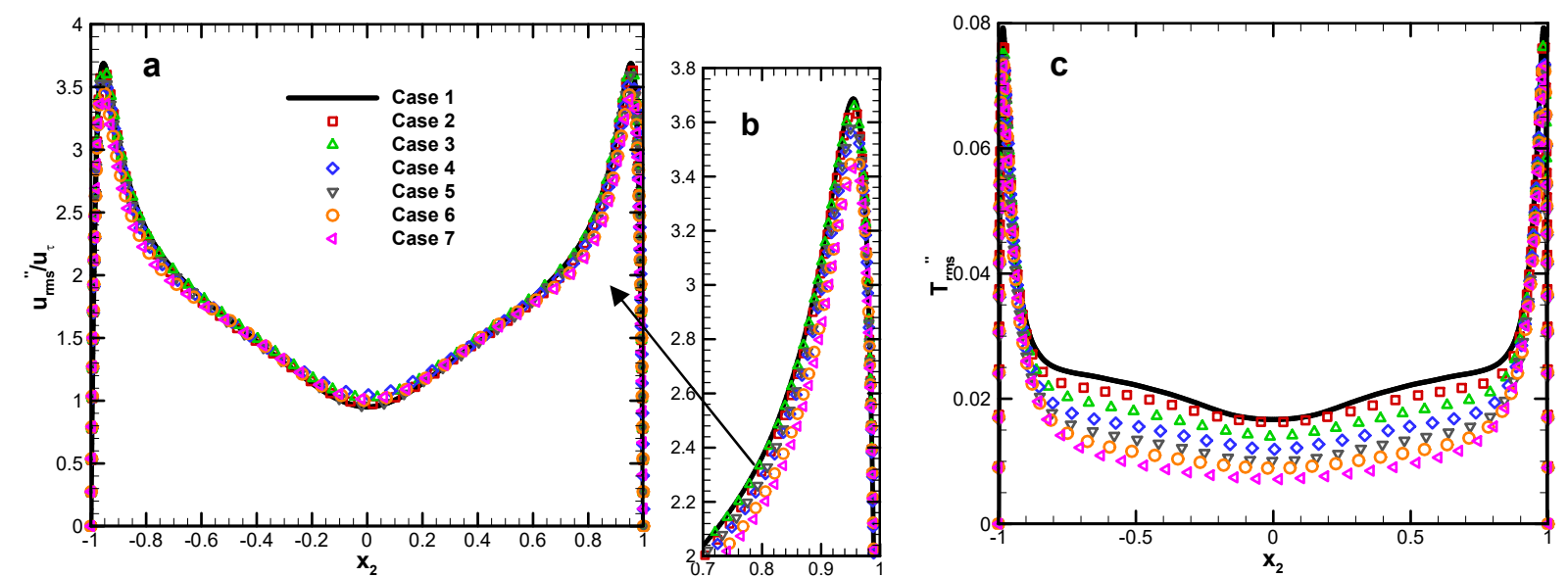

Fig.2 RMS fluctuation of the $a-b)$ streamwise velocity and $c$ ) static temperature.
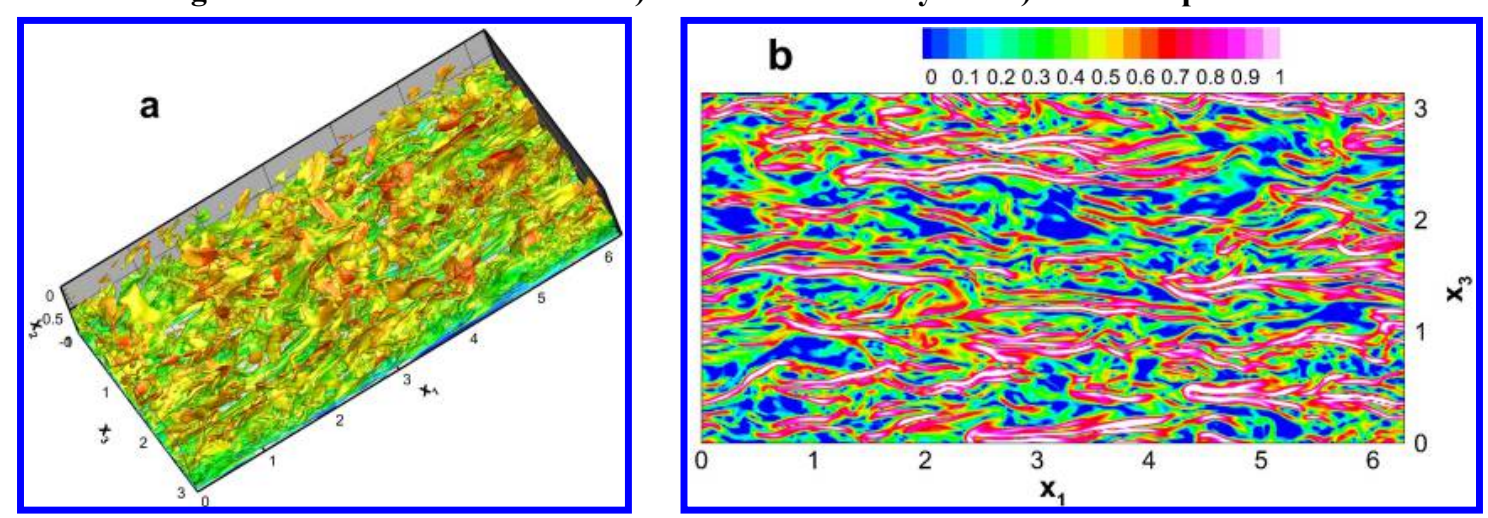

Fig. 3 a) Iso-surfaces of the total temperature gradient magnitude $\left(\log _{10}\left|\nabla T_{t}\right|=0.5\right)$ colored using local static temperature at the same instant for the case $1, b$ ) Instantaneous total temperature gradient magnitude flow field at the section of $\left|x_{2}\right|=0.9$ for the case 1 .

The total temperature, defined as $T_{\mathrm{t}}=T\left(1+(\gamma-1) M a^{2} / 2\right)$, is also one of important parameters for supersonic flow field. To explore the total temperature formation and characteristics, the total temperature gradient magnitude 
$\left(\log _{10}\left|\nabla T_{t}\right|\right)$ exceed prespecified cutoff value are determined. The instantaneous snapshot of case 1 is plotted in fig.3 (a). It is shown that the peak value $\left.\left(\left(\log _{10}\left|\nabla T_{\mathrm{t}}\right|\right)\right)_{\max }\right)$ is 1.528 , and the larger values of $\log _{10}\left|\nabla T_{\mathrm{t}}\right|$ are mainly appeared near the wall. However, when move away from the wall, its value will be reduced gradually. Figure 3 (b) represents the instantaneous snapshots of total temperature gradient magnitude at the section of $x$ - $z$. Similar to the streamwise velocity, several streaks are existed in the spanwise direction, while the mean spanwise spacing of near wall streaks is much smaller. Moreover, the larger value regions of $\log _{10}\left|\nabla T_{\mathrm{t}}\right|$ often appear in pairs. Furthermore, Fig. 4 plots the mean and fluctuating total temperature for different $\mathrm{H}_{2} \mathrm{O}$ mass fraction cases. Like the static temperature distribution, the mean and fluctuating total temperature decrease with $\mathrm{H}_{2} \mathrm{O}$ mass fraction increasing.
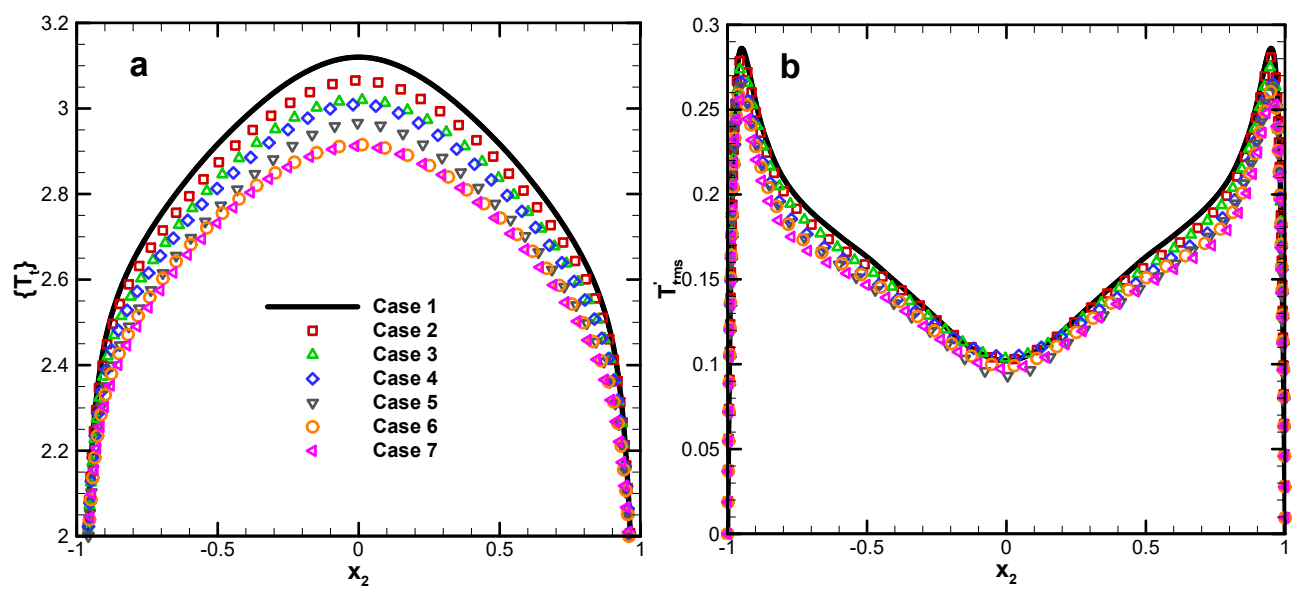

Fig.4 Distribution of the $a$ ) mean total temperature and $b$ ) RMS fluctuation of total temperature.

\section{Velocity-Temperature Correlations}

Figure 5 plots mean-, fluctuating- and turbulent Mach number profiles. The mean Mach number, defined by $\{M a\}=\sqrt{u_{j}^{2}} /\{\mathrm{cc}\}(c c$ is the sound speed), increases to 1 quickly away from the wall, which reveals the main flow is supersonic flow. The turbulent Mach number, defined by $M a_{t}=\sqrt{u_{j}^{\prime 2}} /\{\mathrm{cc}\}$, is an indicator for the significance of compressibility effects. There is excited some regions where $M a_{t}$ larger than 0.3 , particularly near the wall. A more significant increase is observed for the fluctuating Mach number, $M a_{r m s}^{\prime}$, which is the RMS fluctuation of Mach number. An analogical profile exists between the fluctuating and turbulent Mach number, but the former value is smaller than that for the latter among the wall normal direction. 
Due to the channel width is very small, so the boundary layer may fill the entire flow region or its position difficult to confirm. In this paper, the mean streamwise velocity and static temperature at the centerline will be chose to replace that for the boundary layer edge. Figure 6 plots the exact ratios for the DNS data. It can be seen that some deviates are existed as shown in fig.6 (a), which indicate $\mathrm{H}_{2} \mathrm{O}$ mass fraction influence the mean velocity-temperature correlations. To remove the explicit dependence, a new nondimensional parameter $\left(\left(\{T\}-\left\{T_{w}\right\}\right) /\left(\left\{T_{c}\right\}-\left\{T_{w}\right\}\right)\right)$ is introduced. Figure $6(b)$ shows that there is excellent agreement for all $\mathrm{H}_{2} \mathrm{O}$ mass fraction cases.
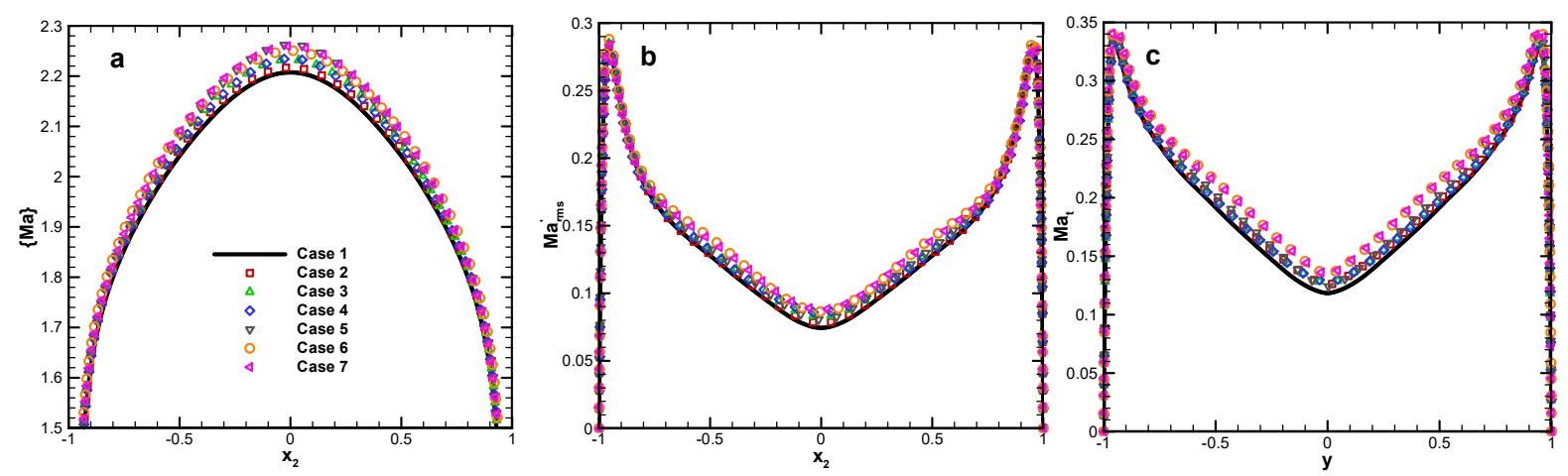

Fig.5 Distribution of the $a$ ) mean Mach number, $b$ ) fluctuating Mach nubmer and $c$ ) turbulent Mach number.
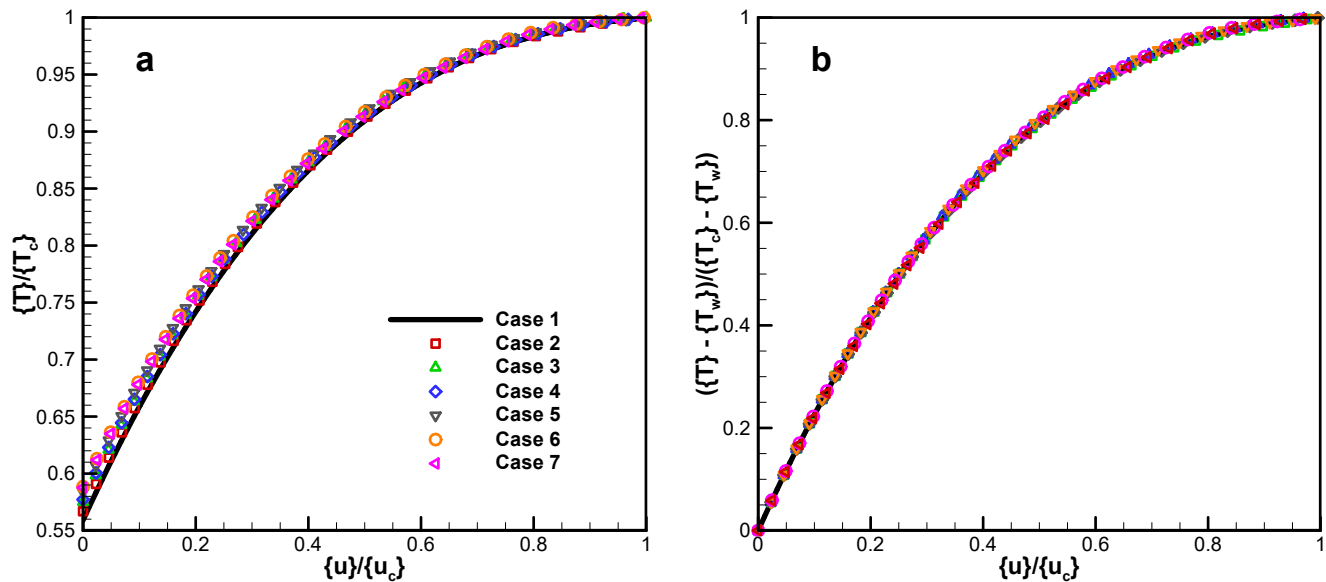

Fig.6 The relationship between velocity $\{u\} / u_{c}$ and temperature $\left.\boldsymbol{a}\right)\{T\} / T_{c}$ and $\left.\boldsymbol{b}\right)\left(\{T\}-T_{w}\right) /\left(T_{c}-T_{w}\right)$.

Morkovin [20] proposed strong Reynolds analogy (SRA) relations, two of which are listed below:

$$
\begin{gathered}
\frac{T_{r m s}^{\prime} /\{T\}}{(\gamma-1) M a^{2}\left(u_{r m s}^{\prime} /\{u\}\right)} \approx 1 \\
R_{u^{\prime} T^{\prime}} \approx-1
\end{gathered}
$$


Figure $7(a)$ plots the relationship between fluctuating streamwise velocity and static temperature, as expressed by (9), for supersonic turbulent channel flows. It is shown that the agreement is not perfect with all $\mathrm{H}_{2} \mathrm{O}$ mass fraction cases. Similar results have been reported by Morinishi et al. [16]. They explained that not only the total temperature fluctuation can't be negligible compared to the static temperature fluctuation, but also the condition of $\left\langle T^{\prime 2}\right\rangle /\{T\}^{2} \ll\left(\left\langle T_{t}^{\prime 2}\right\rangle-2\left\langle T^{\prime} T_{t}^{\prime}\right\rangle /\{T\}^{2}\right)$ is not satisfied. Figure $7(b)$ shows that better agreement is achieved between current DNS results except for $1.0<|\mathrm{y}|<0.6$, and the modified strong Reynolds analogy (ESRA) of Cebeci et al. [21], which is given by:

$$
\frac{T_{r m s}^{\prime} /\{T\}}{(\gamma-1) M a^{2}\left(u_{r m s}^{\prime} /\{u\}\right)} \approx\left[1+C p \frac{\left\{T_{w}\right\}-\left\{T_{t, c}\right\}}{\{u\}\left\{u_{c}\right\}}\right]
$$

As shown in Fig.7 (c), the correlation $R_{u^{\prime}} T^{\prime}$ is negative through the channel, which indicates that $u$ ' and $T$ ' have a negative correlation. Moreover, the above correlations near to -1 in the near wall, and such dependent value quickly degrade in the largest region that is far away from the wall. It is shown that $R_{u}{ }^{\prime}$, is a strong function of $\mathrm{H}_{2} \mathrm{O}$ mass fraction, particularly in the flow field. The correlation dependent value will be enhanced with $\mathrm{H}_{2} \mathrm{O}$ mass fraction increasing.
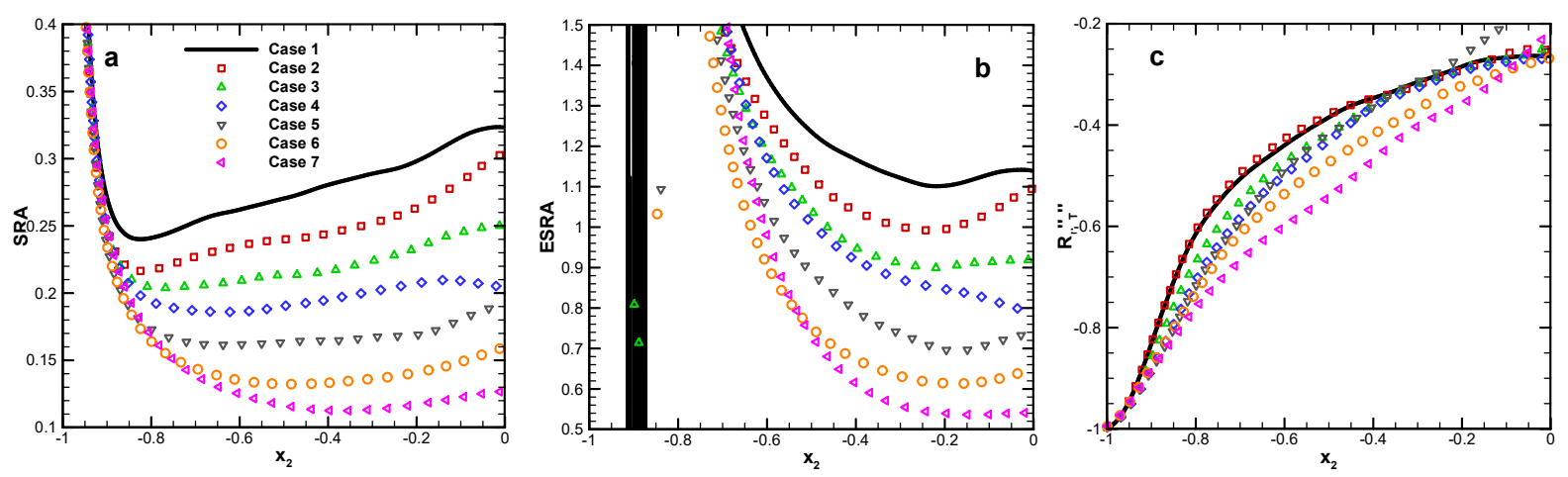

Fig.7 Distribution of the a) SRA, b) ESRA and c) $R_{u}$ ' $T^{*}$

\section{Conclusion}

In this paper, using $7^{\text {th }}$ order WENO scheme combined with $8^{\text {th }}$ order central scheme, we have performed DNS of supersonic turbulent channel flows. Mach number, based on the bulk velocity and sound speed at the walls, of 2.56 is considered; Reynolds number, defined in terms of the bulk velocity and channel half width, are of the order of 7000; water vapor $\left(\mathrm{H}_{2} \mathrm{O}\right)$ mass fraction are from 0.000 to 0.161 . The velocity and temperature statistical 
characteristics and velocity-temperature correlations have been analyzed, and some conclusions can be drawn as follows:

It is shown that many of turbulent statistical characteristics used to express supersonic turbulent channel flow of pure air also hold for the $\mathrm{H}_{2} \mathrm{O}$ considered. In particular, when $\mathrm{H}_{2} \mathrm{O}$ mass fraction is increased, the mean and fluctuating streamwise velocity are few changing, while the specific heat, static temperature and total temperature are decreased. Simultaneously, the turbulence Mach number (turbulent Mach number, fluctuating Mach number) are increased dramatically.

It is found that $\mathrm{H}_{2} \mathrm{O}$ mass fraction influence the mean velocity-temperature correlations. A nondimensional parameter, which is a relative static temperature ratio formula, can be used to connect the mean static temperature with streamwise velocity. In terms of strong Reynolds analogy (SRA), the original SRA relation breaks down for all cases. The Cebeci's version of the modified SRA provides better agreement. In addition, the correlation $R_{u} T^{\prime}$, isn't remained the same between the different $\mathrm{H}_{2} \mathrm{O}$ mass fraction cases.

\section{Acknowledgments}

This work is performed with the support and under the auspices of the National Natural Science Foundation of China (No. 11502236), Natural Science Foundation of Zhejiang Province (No. LQ16E090005), the Open Research Subject of Key Laboratory of Pump and Motor of Zhejiang Province (No. H2015G001) and the Science Foundation of Zhejiang Sci-Tech University (No. 11130032611303).

\section{References}

[1] Pellert, G. L., Bruno, C., and Chinitz, W., Air Vitiation Effects on Scramjet Combustion Tests, RTO-TR-AVT-007-V2, 2006.

-[2] Edelman, R. B., and Spadaccini, L. J., "Theoretical Effects of Vitiated Air Contamination on Ground Testing of Hypersonic Air Breathing Engines,” Journal of Spacecraft and Rockets, Vol. 6, No. 12, 1969, pp. 1442-1447.

[3] Le, J. L., Liu, W. X., and Song, W. Y., "Experimental and Numerical Investigation of Air Vitiation Effects on Scramjet Test Performance," AIAA Paper, 2009-7344, 2009.

[4] Mitani, T., "Ignition Problems in Scramjet Testing," Combustion and Flame, Vol. 101, No. 3, 1995, pp. 347-359.

-[5] Tohru, M., Tetsuo, H., and Shigeru, S., "Comparison of Scramjet Engine Performance in Mach 6 Vitiated and Storage-Heated Air," Journal of Propulsion and Power, Vol. 13 No. 5, 1997, pp. 635-642.

[6] Lai, H. T., and Thomas, S. R., "Numerical Study of Contaminant Effects on Combustion of Hydrogen, Ethane, and Methane in Air," AIAA Paper, 1995-6097, 1995. 
[7] Huang, W., Wang, Z. G., and Li, S. B., "Influences of $\mathrm{H}_{2} \mathrm{O}$ Mass Fraction and Chemical Kinetics Mechanism on the Turbulent Diffusion Combustion of $\mathrm{H}_{2}-\mathrm{O}_{2}$ in Supersonic Flows,” Acta Astronautica, Vol. 76, 2012, pp. 51-59.

- [8] Gokulakrishnan, P., Fuller, C. C., and Klassen, M. S., "Experiments and Modeling of Propane Combustion with Vitiation," Combustion and Flame, Vol. 161, 2014, pp. 2038-2053.

[9] Pellett, G. L., Bruno, C., and Chinitz, W., "Review of Air Vitiation Effects on Scramjet Ignition and Flame Holding Combustion Processes,” AIAA Paper, 2002-3880, 2002.

- [10] Ingenito, A., "Theoretical Investigation of Air Vitiation Effects on Hydrogen Fuelled Scramjet Performance," International Journal of Hydrogen Energy, Vol. 40, 2015, pp. 2862-2870.

-[11] Coleman, G. N., Kim, J., and Moser, R. D., “A numerical study of Turbulent Supersonic Isothermal-Wall Channel Flow,” Journal of Fluid Mechanics, Vol. 305, 1995, pp. 159-183.

[12] Huang, P. G., Coleman, G. N., and Bradshaw, P., "Compressible Turbulent Channel Flows: DNS Results and Modeling," Journal of Fluid Mechanics, Vol. 305, 1995, pp. 185-218.

[13] Tamano, S., and Morinishi, Y., "Effect of Different Thermal Wall Boundary Conditions on Compressible Turbulent Channel Flow at M=1.5,” Journal of Fluid Mechanics, Vol. 548, 2006, pp. 361-373.

-[14] Duan, L., and Martin, M. P., "Direct Numerical Simulation of Hypersonic Turbulent Boundary Layers. Part 2: Effect of Wall Temperature,” Journal of Fluid Mechanics, Vol. 655, 2010, pp. 419-445.

[15] Liang, X., and Li, X. L., "DNS of a Spatially Evolving Hypersonic Turbulent Boundary Layer at Mach 8," Scientia Sinica Physica, Mechanica \& Astronomica, Vol. 56, 2013, pp. 1408-1418.

-[16] Morinishi, Y., Tamano, S., and Nakabayashi, K., "Direct Numerical Simulation of Compressible Turbulent Channel Flow between Adiabatic and Isothermal Walls,” Journal of Fluid Mechanics, Vol. 502, 2004, pp. 273-308.

[17] Duan, L., and Martin, M. P., "Direct Numerical Simulation of Hypersonic Turbulent Boundary Layers. Part 3: Effect of Mach number," Journal of Fluid Mechanics, Vol. 672, 2011, pp. 245-267.

[18] Duan, L., and Martin, M. P., "Direct Numerical Simulation of Hypersonic Turbulent Boundary Layers. Part 4: Effect of High Enthalpy,” Journal of Fluid Mechanics, Vol. 684, 2011, pp. 25-59.

- [19] Chen, X. P., and Li, X. L., "Direct Numerical Simulation of Chemical Nonequilibrium Turbulent Flow," Chinese Physics Letters, Vol. 30, No. 6, 2013, 064702.

[20] Morkovin, M. V., "Effects of Compressibility on Turbulent Flows," In Mechanique de la Turbulence (ed. A. Favre), 1964, pp. 367-380.

[21] Cebeci, T., and Smith, A. M. O., Analysis of Turbulent Boundary Layers, Academic Press, New York, 1974, Chaps. 4. 
[22] Chen, X. P., Dou, H.-S., “Application of Direct Numerical Simulation Method to High Reynolds Number Turbulent Channel Flow,” 2015 International Conference on Mechanical and Aeronautical Engineering (ICMAE 2015), December 11-14, 2015, Singapore.

[23] Mcbride, B. J., and Zehe, M. J., "NASA Glenn Coefficients for Calculating Thermodynamic Properties of Individual Species,” NASA TP-2002-211556, 2002.

[24] CFD-ACE+ Modules Manual, Part 1, 2010.

[25] Chase, M. W., "JANAF Thermo-Chemical Tables,” $4^{\text {nd }}$ ed., Journal Physical and Chemical Reference Data, 20899-0001, 1998.

[26] Barbante, P. F., and Magin, T. E., "Critical Technologies for Hypersonic Vehicle Development: Fundamentals of Hypersonic Flight- Properties of High Temperature Gases,” North Atlantic Treaty Organisation, RTO-EN-AVT-116, 2004.

-[27] Jiang, G. S., and Shu, C. W., "Efficient Implementation of Weighted ENO Schemes,” Journal Computations Physics, Vol. 126, 1996, pp. 202-222. 